\title{
Assessment of MR stereotactic imaging and image co-registration accuracy for 3 different MR scanners by 3 different methods/phantoms: phantom and patient study
}

\author{
Veronika Paštyková, MSc, ${ }^{1}$ Josef Novotný Jr., PhD, ${ }^{1}$ Tomáš Veselský, PhD, ${ }^{1}$ \\ Dušan Urgošík, MD, PhD, ${ }^{2}$ Roman Liščák, MD, PhD, ${ }^{2}$ and Josef Vymazal, MD, DSc ${ }^{3}$ \\ Departments of ${ }^{1}$ Medical Physics, ${ }^{2}$ Stereotactic and Radiation Neurosurgery, and ${ }^{3}$ Radiodiagnostics, Na Homolce Hospital, \\ Prague, Czech Republic
}

\begin{abstract}
OBJECTIVE The aim of this study was to compare 3 different methods to assess the geometrical distortion of two 1.5$T$ and one 3-T magnetic resonance (MR) scanners and to evaluate co-registration accuracy. The overall uncertainty of each particular method was also evaluated.

METHODS Three different MR phantoms were used: 2 commercial CIRS skull phantoms and PTGR known target phantom and 1 custom cylindrical Perspex phantom made in-house. All phantoms were fixed in the Leksell stereotactic frame and examined by a Siemens Somatom CT unit, two 1.5-T Siemens (Avanto and Symphony) MRI systems, and one 3-T Siemens (Skyra) MRI system. The images were evaluated using Leksell GammaPlan software, and geometrical deviation of the selected points from the reference values were determined. The deviations were further investigated for both definitions including fiducial-based and co-registration-based in the case of the CIRS phantom images. The same co-registration accuracy assessment was also performed for a clinical case. Patient stereotactic imaging was done on 3-T Skyra, 1.5-T Avanto, and CT scanners.
\end{abstract}

RESULTS The accuracy of the CT scanner was determined as $0.10,0.30$, and $0.30 \mathrm{~mm}$ for $X, Y$, and $Z$ coordinates, respectively. The total estimated uncertainty in distortion measurement in one coordinate was determined to be $0.32 \mathrm{~mm}$ and $0.14 \mathrm{~mm}$, respectively, for methods using and not using CT as reference imaging. Slightly more significant distortions were observed when using the 3-T than either 1.5-T MR units. However, all scanners were comparable within the estimated measurement error. Observed deviation/distortion for individual $X, Y$, and $Z$ stereotactic coordinates was typically within $0.50 \mathrm{~mm}$ for all 3 scanners and all 3 measurement methods employed. The total radial deviation/distortion was typically within $1.00 \mathrm{~mm}$. Maximum total radial distortion was observed when the CIRS phantom was used; $1.08 \pm 0.49$ $\mathrm{mm}, 1.15 \pm 0.48 \mathrm{~mm}$, and $1.35 \pm 0.49 \mathrm{~mm}$ for Symphony, Avanto, and Skyra, respectively. The co-registration process improved image stereotactic definition in a clinical case in which fiducial-based stereotactic definition was not accurate; this was demonstrated for 3-T stereotactic imaging in this study. The best results were shown for 3-T MR image coregistration with $\mathrm{CT}$ images improving image stereotactic definition by about $0.50 \mathrm{~mm}$. The results obtained with patient data provided a similar trend of improvement in stereotactic definition by co-registration.

CONCLUSIONS All 3 methods/phantoms used were evaluated as satisfactory for the image distortion measurement. The method using the PTGR phantom had the lowest uncertainty as no reference CT imaging was needed. Image coregistration can improve stereotactic image definition when fiducial-based definition is not accurate.

https://thejns.org/doi/abs/10.3171/2018.7.GKS181527

KEYWORDS stereotactic MR imaging; image co-registration; phantom measurement; stereotactic methods; stereotactic radiosurgery

$\mathrm{T}$ HE use of modern stereotactic methods has increased the requirements for precise stereotactic imaging. Imaging techniques such as computed tomography (CT) and magnetic resonance imaging (MRI) are routinely used for target localization in stereotactic radiosurgery.
The choice of imaging technique depends on various factors, including individual radiological lesion parameters as well as patient status and medical history (e.g., surgical implants, pace makers, shunts, etc.). It is commonly accepted that CT imaging provides more precise geometri-

ABBREVIATIONS CT = computed tomography; MRI = magnetic resonance imaging; PET = positron emission tomography.

SUBMITTED May 30, 2018. ACCEPTED July 31, 2018.

INCLUDE WHEN CITING DOI: 10.3171/2018.7.GKS181527. 
cal localization with less contrast resolution of anatomical structures and the lesion itself, ${ }^{27,28}$ while MRI provides better resolution of many radiosurgical lesions. In addition, MRI can be performed in any plane, including the axial, coronal, and sagittal planes. ${ }^{28}$ The accuracy of the delivered dose by Leksell Gamma Knife (LGK) or any other radiosurgical techniques strongly depends on the precision of the MR images used for treatment planning. The use of MRI for stereotactic treatment has, unfortunately, 2 shortcomings; the electron density of the tissue is not provided for dosimetric (heterogeneity) calculation, and geometrical distortion of the images can be observed. However, the bulk electron density can be assigned to the structure contour for brain MRI when heterogeneity correction for the dose calculation is necessary. ${ }^{20,26}$

MRI geometrical distortion is a complex issue and can have various causes. To obtain data, the static B0 magnetic field, radiofrequency B1 field, and 3 independent linear gradient magnetic fields are used. When the static B0 field and ideal gradient fields are perturbed, image distortion can occur. ${ }^{15,24}$ The possible causes of the distortion can be defined as hardware related or tissue related. The main sources contributing to hardware-related distortions are inhomogeneity of the main magnetic field as well as nonlinearity of the gradient fields, but they can also be related to switching of the gradient coils. On the other hand, tissue-related distortions are caused mainly by chemical shift effects. Focusing on distortions caused by gradient field nonlinearities, these can manifest in 3 ways: barrel aberration, potato-chip effects, and bow-tie effects. The only type of geometrical distortion produced in 3D MRI is the barrel aberration. ${ }^{26}$

It is also necessary to note that newer MRI technology does not automatically imply better MR image geometrical accuracy. It must be considered that the geometrical distortions depend on the MR model/unit itself, slice orientation, scanning parameters, scanning position from the center of the head coil, and certain materials related to the patient (surgical clips, dental materials, etc.). Geometrical distortions vary between scanning protocols even on the same MR scanner, and it is essential to consider the MRI setup and scanning protocols. Parameters that can help include applying whisper gradients mode, nonselective excitation, optimal bandwidth, and distortion correction if available. Typically, more massive geometrical distortions are observed with coronal orientation than with axial orientation. Greater distortions are also seen in noncentrally located slice positions in the investigated volume (further inferior or further superior). ${ }^{14,15}$

The complex solution to check MRI distortion consists of measurements of the fiducial geometry individually for every patient, comparison of images from both CT and MRI, and, most importantly, phantom measurements. Several authors have evaluated geometrical distortion of MR images and performed measurements with the phantoms. ${ }^{11-13,16,18,23}$ However, uncertainties involved in imaging and image definition processes still need to be discussed. Most distortion correction techniques consist of 3 general components: identification and registration of a fixed implemented coordinate system, which is derived from the reference data set. The next steps are to quantify the distortions and apply the distortion correction algorithm. To measure the distortion accurately, phantoms with the established geometry must be used, usually consisting of square grids, cylindrical rods, or capillary tubes. ${ }^{17,28}$

Image co-registration is the process of finding the mathematical transformation that aligns several different radiographic studies. ${ }^{4}$ Co-registration software has been implemented to the latest versions of Leksell GammaPlan (LGP). There are obvious potential advantages of including image co-registration processes in treatment planning, especially for preplanning or patient follow-up. The patient can be imaged by nonstereotactic MRI, and the co-registration process may be used in combination with stereotactic imaging by MRI or CT for treatment or post-treatment monitoring. ${ }^{4,6,13}$ It is possible to use it also when the stereotactic definition cannot be performed (for example, due to missing fiducials in the image). Co-registration of the MR images with the CT scans can also improve image stereotactic definition accuracy when the primary method based on fiducial definition is not satisfactory for the stereotactic treatment purposes due to the high fiducial errors.

Although many studies have evaluated MR image distortions by several different phantoms and methods, very limited information can be found about the uncertainty of the measurement itself. Many studies report submillimeter results when evaluating MRI distortions but no information regarding the uncertainty of these measurements. The aim of this study was to perform assessment and comparison of MRI distortions with the use of 3 different phantoms and methods ( 2 commercial and 1 made in-house). The second aim was to validate the uncertainty in image co-registration and determine whether image coregistration can improve stereotactic image definition, especially in cases when the primary method (fiducial-based image definition) fails. Image distortion was evaluated and compared for 3 different Siemens MRI scanners, two 1.5 $\mathrm{T}$ and one $3 \mathrm{~T}$.

\section{Methods}

Three different phantoms (PTGR, CIRS, and custommade) were used in this study. The PTGR phantom (Physikalisch-Technische Gesellschaft für Radiologie $\mathrm{GmbH}$ ) consists of slabs with 21 embedded known targets (crosshairs with plastic vials, $3 \mathrm{~mm}$ outside diameter, $1.5 \mathrm{~mm}$ inside diameter). Crosshairs are positioned at known Leksell coordinates and cover the whole stereotactic space. ${ }^{10,19}$ Crosshairs can be filled with contrast medium for MR imaging; in this case, the same $\mathrm{CuSO}_{4}$ (copper sulfate) solution as for the stereotactic imaging box was used. The rest of the tissue-equivalent plastic phantom is filled with water. Since stereotactic coordinates of 21 crosshairs are known from manufacturers (when the phantom is fixed in a Leksell stereotactic frame), it is not necessary to perform any reference imaging.

The next phantom was the CIRS (Computer Imaging and Reference Systems, Inc.) 3D anthropomorphic skull phantom model 603A. The whole cranial portion of the skull volume is filled with a 3D matrix of 3-mm-diameter rods spaced $15 \mathrm{~mm}$ apart. It was designed for end-to-end testing, and thanks to the skull-like filling can be imaged 


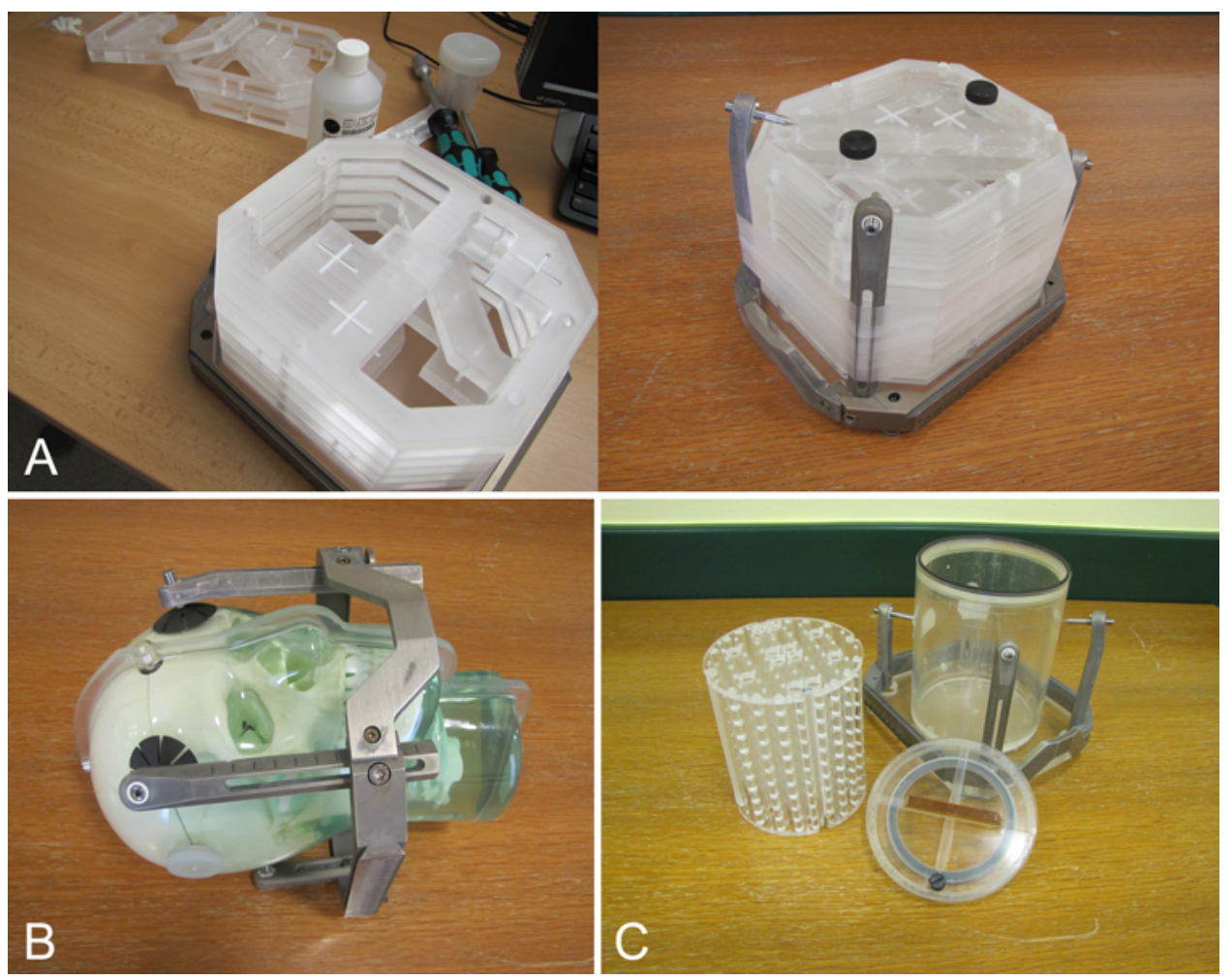

FIG. 1. Phantoms used for measurements. A: PTGR known target phantom consisting of 8 slabs with the 21 contrast mediumfilled crosshairs. B: CIRS anthropomorphic skull phantom. C: Custom-made cylindrical Perspex phantom with an insert consisting of solid plastic rods.

using most modalities, including $\mathrm{CT}$ and various MRI sequences. ${ }^{2}$ The individual pads of the phantom allow it to be fixated in the stereotactic frame and used for the evaluation of the geometrical distortion during imaging or image co-registration. Reference imaging (e.g., CT) is needed for this phantom when evaluating MRI distortion.

The custom-made cylindrical Perspex phantom contains an insert consisting of solid plastic rods (3-mm diameter, spaced $15 \mathrm{~mm}$ apart). There are 55 and 72 plastic rods in 2 orientations positioned in a single insert. The rest of the phantom is filled with water. ${ }^{15}$ Reference imaging (e.g., $\mathrm{CT}$ ) is needed for this phantom when evaluating MRI distortion. The design of all 3 phantoms can be seen in Fig. 1.

All 3 phantoms were mounted in the Leksell stereotactic head frame for standard patient treatment and investigated within the MRI head coil. All 3 phantoms used were imaged by CT and MRI scanners with the Leksell fiducial box attached. For CT imaging, a Siemens Somatom Definition Flash was used, and for MRI, a Siemens 1.5-T Magnetom Symphony, 1.5-T Magnetom Avanto, and 3-T Magnetom Skyra were used.

Identical scanning conditions as for patient imaging were used; the same stereotactic head frame, screws, and MRI adaptor and identical positioning. The PTGR and the custom-made cylindrical Perspex phantoms could not be mounted with the use of frame posts and pins as in the case of a patient, the torque of the pins causing potentially small mechanical distortion of the frame was thus excluded in this study. However, posts and pins were always included in all 3 phantoms as a potential source of an MRI distortion. We believe the mechanical frame distortion should be generally very small and detectable by attaching an MRI Leksell fiducial box. T1-weighted 3D imaging was performed using a whisper gradient echo. The complete list of scanning parameters used for all 3 scanners is shown in Table 1. The parameters of the scan-

TABLE 1. Acquisition parameters for Siemens CT and MRI

\begin{tabular}{lcccc}
\hline \multicolumn{1}{c}{ Parameter } & $\begin{array}{c}\text { CT } \\
\text { Somatom }\end{array}$ & $\begin{array}{c}\text { Avanto } \\
1.5 \mathrm{~T}\end{array}$ & $\begin{array}{c}\text { Symphony } \\
1.5 \mathrm{~T}\end{array}$ & $\begin{array}{c}\text { Skyra } \\
3 \mathrm{~T}\end{array}$ \\
\hline Voltage $(\mathrm{V})$ & 120 & - & - & - \\
\hline Current (mA) & 205 & - & - & - \\
\hline Matrix size & $512 \times 512$ & $256 \times 256$ & $179 \times 256$ & $173 \times 256$ \\
\hline No. of slices & 140 & 176 & 144 & 144 \\
\hline Slice thickness (mm) & 1.00 & 1.00 & 1.25 & 1.00 \\
\hline Slice gap (mm) & 0.00 & 0.00 & 0.00 & 0.00 \\
\hline Field of view (mm) & 240 & 256 & 250 & 256 \\
\hline Image mode & - & $3 \mathrm{D} \mathrm{TFL}$ & $3 \mathrm{D} \mathrm{FL}$ & $3 \mathrm{D} \mathrm{FL}$ \\
\hline Encoding & - & $\mathrm{Rt}-\mathrm{Lt}$ & $\mathrm{Rt}-\mathrm{Lt}$ & $\mathrm{Rt}-\mathrm{Lt}$ \\
\hline Repetition time & - & 2030 & 25 & 16 \\
$\quad$ (msec) & - & & & \\
\hline Echo time (msec) & - & 3.54 & 3.1 & 4.32 \\
\hline No. of excitations & - & 1 & 1 & 1 \\
\hline
\end{tabular}


TABLE 2. Assessment of CT scanner accuracy by PTGR phantom

\begin{tabular}{cc}
\hline & Mean Deviation in $\mathrm{mm}$ \\
\hline$\Delta \mathrm{X}$ & 0.10 \\
\hline$\Delta \mathrm{Y}$ & 0.30 \\
\hline$\Delta \mathrm{Z}$ & 0.30 \\
\hline$\Delta \mathrm{R}$ & $0.47 \pm 0.19$ \\
\hline
\end{tabular}

ning sequences correspond to the standard patient scanning conditions, and the setting was chosen to approach the standard situation as much as possible.

It was possible to apply distortion correction provided by Siemens as a part of an operator's software used for preparing and controlling the scanning process when imaging on the Avanto and Skyra MRI scanners. The final images were processed in Leksell GammaPlan software and defined based on fiducial markers. First, the PTGR phantom was used for the evaluation of the CT imaging inaccuracy - the stereotactic coordinates of 21 points were compared with manufacturer-quoted values. The inaccuracy of reference imaging methods (CT) for CIRS and custom-made phantoms was thus determined.

The MR images of all 3 phantoms were first registered based on fiducial markers, and deviations from the chosen point's position (stereotactic coordinate) on the CT versus MR images were determined. For the PTGR phantom, the deviations from the manufacturer-quoted values of the reference points were determined the same way as for CT scanning. The deviations between stereotactic coordinates based on CT and MRI were evaluated in the case of CIRS and custom-made phantoms.

The coordinates were separately measured in Leksell GammaPlan software. Deviations in 3 stereotactic individual coordinates $\Delta \mathrm{X}, \Delta \mathrm{Y}$, and $\Delta \mathrm{Z}$ were determined, and the total deviation $\Delta \mathrm{R}$ was calculated as

$$
\Delta \mathrm{R}=\sqrt{\Delta \mathrm{X}^{2}+\Delta \mathrm{Y}^{2}+\Delta \mathrm{Z}^{2}} .
$$

Because the CIRS anthropomorphic phantom is the closest to patient head dimensions and shape, and also provides some anatomical structures, it was used for image co-registration assessment. In order to identify whether there is a benefit to image co-registration versus fiducial-based stereotactic definition, MRI with the largest distortions was selected on the basis of the stereotactic coordinate deviation data for the 3 evaluated MRI scanners (see Table 4). The largest deviations were observed for the 3-T Skyra scanner. Co-registration was performed using the implemented software in Leksell GammaPlan, and 3-T Skyra images were co-registered subsequently to stereotactic images from 1.5-T Symphony, 1.5-T Avanto, and CT. The same 30 point positions as in the previous measurement were read out, and the deviations from the reference $\mathrm{CT}$ points evaluated. As a reference image, CT was chosen in order to evaluate the benefit of image coregistration.

The same co-registration accuracy assessment was also performed for a clinical case. Patient stereotactic imaging was done on 3-T Skyra, 1.5-T Avanto, and CT scanner.
TABLE 3. Estimated total uncertainty of measurement

\begin{tabular}{lccc}
\hline & \multicolumn{3}{c}{$\begin{array}{c}\text { Estimated Uncertainty of } \\
\text { Measurement in mm }\end{array}$} \\
\cline { 2 - 4 } Uncertainty & Custom & PTGR & CIRS \\
\hline $\begin{array}{l}\text { Mechanical accuracy of phantom } \\
\text { manufacturing }\end{array}$ & NA (0.10) & 0.10 & NA $(0.10)$ \\
\hline Accuracy of CT reference image & 0.30 & NA & 0.30 \\
\hline $\begin{array}{l}\text { Precision of point measurement in } \\
\text { Leksell GammaPlan }\end{array}$ & 0.10 & 0.10 & 0.10 \\
\hline Total uncertainty & 0.32 & 0.14 & 0.32 \\
\hline NA = not applicable. & & & \\
\end{tabular}

Images from the 3-T Skyra were first defined based on fiducial markers and then subsequently co-registered to 1.5-T Avanto and CT stereotactic template images. As a reference, CT images were taken. Co-registration was also performed by using the implemented software in Leksell GammaPlan as for the phantoms. The stereotactic coordinates $\mathrm{X}, \mathrm{Y}$, and $\mathrm{Z}$ were measured for 10 various anatomical structures subsequently in CT fiducial-based stereotactic images, 3-T Skyra fiducial-based stereotactic images, 3-T Skyra co-registered images to stereotactic 1.5-T Avanto images, and 3-T Skyra co-registered images to stereotactic CT images. The following 10 anatomical structures were measured: 1) pontomesencephalic vein running along the trigeminal nerve, 2) basilar artery at the pontomesencephalic junction (bifurcation between the posterior cerebral arteries), 3) tentorial roof, 4) anterior commissure, 5) proximal part of the infundibulum, 6) basilar artery at the level of the inferior sella turcica, 7) tentorial roof (second point), 8) anterior horn of the right lateral ventricle, 9) anterior horn of the left lateral ventricle, and 10) anterior portion of a pseudocyst in the left thalamus. The deviations in 3 stereotactic individual coordinates $\Delta \mathrm{X}, \Delta \mathrm{Y}$, and $\Delta \mathrm{Z}$ were determined (with CT taken as a reference imaging) as well as the total deviation $\Delta \mathrm{R}$ for both phantom and patient measurements. Although we are aware of the potential uncertainty in choosing anatomical points/landmarks for reference points of measurement, it is equilibrated by using and demonstrating results on data from a real patient (and not a phantom). Our main purpose in using real clinical data was to confirm the trend observed with phantoms.

\section{Results}

In order to assess the uncertainty of the CIRS and custom-made phantom-based method of MRI geometrical distortion measurement, CT stereotactic imaging accuracy was evaluated with the PTGR phantom. The results are shown in Table 2. The precision of point measurement in Leksell GammaPlan was evaluated by 2 independent observers for 10 consecutive readings of stereotactic coordinates by each observer for an identical point with 3 different phantoms. The uncertainty in choosing points as well as the anatomical landmarks was determined. The results of CT scanner accuracy measurements together with the uncertainty of separate point readout and mechanical accuracy of phantom manufacturing were used 
TABLE 4. Individual $\Delta \mathrm{X}, \Delta \mathrm{Y}$, and $\Delta \mathrm{Z}$ stereotactic coordinate deviations and total $\Delta \mathrm{R}$ deviation for all 3 phantoms and 3 evaluated MRI scanners

\begin{tabular}{|c|c|c|c|c|c|c|c|c|c|c|c|c|}
\hline \multirow[b]{2}{*}{ Scanner } & \multicolumn{4}{|c|}{ Custom } & \multicolumn{4}{|c|}{ PTGR } & \multicolumn{4}{|c|}{ CIRS } \\
\hline & $\Delta \mathrm{X}$ & $\Delta \mathrm{Y}$ & $\Delta Z$ & $\Delta \mathrm{R}$ & $\Delta \mathrm{X}$ & $\Delta \mathrm{Y}$ & $\Delta \mathrm{Z}$ & $\Delta \mathrm{R}$ & $\Delta \mathrm{X}$ & $\Delta \mathrm{Y}$ & $\Delta Z$ & $\Delta \mathrm{R}$ \\
\hline Symphony $1.5 \mathrm{~T}$ & 0.25 & 0.63 & 0.45 & $0.90 \pm 0.31$ & 0.19 & 0.34 & 0.60 & $0.78 \pm 0.47$ & 0.22 & 0.54 & 0.76 & $1.08 \pm 0.49$ \\
\hline Avanto $1.5 \mathrm{~T}$ & 0.26 & 0.40 & 0.30 & $0.63 \pm 0.23$ & 0.28 & 0.67 & 0.38 & $0.92 \pm 0.39$ & 0.20 & 0.42 & 0.97 & $1.15 \pm 0.48$ \\
\hline Skyra 3 T & 0.27 & 0.48 & 0.42 & $0.78 \pm 0.37$ & 0.25 & 0.75 & 0.65 & $1.20 \pm 0.53$ & 0.27 & 0.68 & 1.03 & $1.35 \pm 0.49$ \\
\hline
\end{tabular}

Data are presented as the mean deviation in millimeters ( \pm SD for $\Delta R$ ).

for calculation of the total uncertainty. An overview of the uncertainties included in the total uncertainty calculation is presented in Table 3.

The X, Y, and Z stereotactic coordinates of grid points in each phantom were read out in Leksell GammaPlan software in order to obtain $\Delta \mathrm{X}, \Delta \mathrm{Y}$, and $\Delta \mathrm{Z}$ as a deviation between the manufacturer's values (for the PTGR phantom) and CT-based values (for the CIRS and custom-made phantoms). There were 21 points for the PTGR phantom,
30 points for the CIRS phantom, and 48 points for the custom-made phantom evaluated throughout stereotactic space. Individual $\Delta \mathrm{X}, \Delta \mathrm{Y}$, and $\Delta \mathrm{Z}$ stereotactic coordinate deviations and total $\Delta \mathrm{R}$ deviation for all 3 phantoms and 3 evaluated MRI scanners are shown in Table 4. Images of all 3 phantoms as imported and evaluated in Leksell GammaPlan are shown in Fig. 2.

The CIRS phantom co-registration deviations were evaluated in the same 30 points as for the fiducial-based
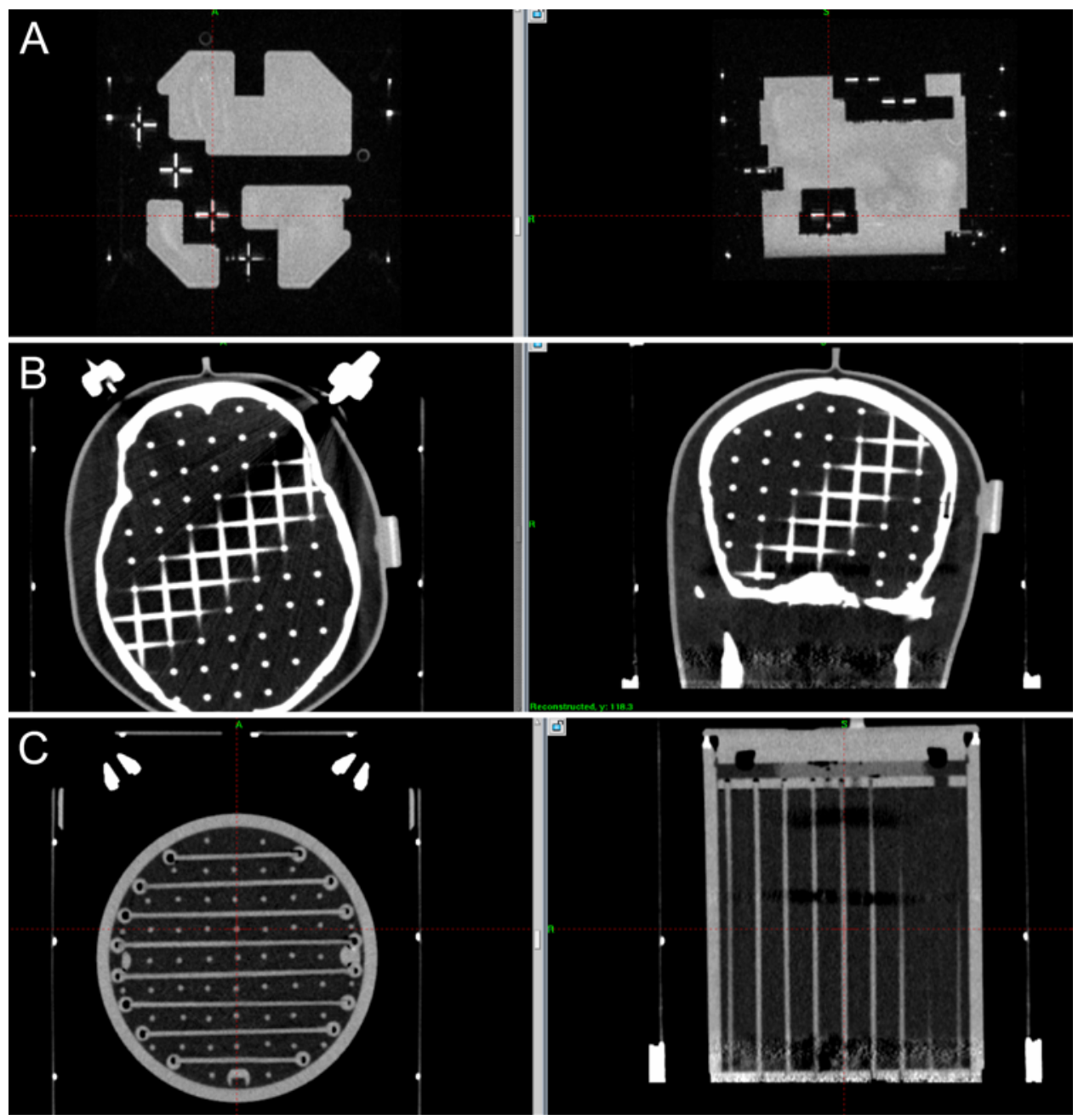

FIG. 2. Axial (left) and coronal (right) images of all 3 phantoms as imported and evaluated in Leksell GammaPlan. A: PTGR phantom. B: CIRS phantom. C: Custom-made phantom. The phantom design, including grid points, can be seen. 
TABLE 5. Results of image co-registration for the CIRS phantom

\begin{tabular}{|c|c|c|c|c|}
\hline \multirow{2}{*}{$\begin{array}{l}\text { Phantom Data } \\
\text { Co-registration }\end{array}$} & \multicolumn{4}{|c|}{$\begin{array}{l}\text { Image Co-Registration Mean } \\
\text { Deviation in } \mathrm{mm}\end{array}$} \\
\hline & $\Delta \mathrm{X}$ & $\Delta \mathrm{Y}$ & $\Delta \mathrm{Z}$ & $\Delta \mathrm{R}$ \\
\hline MR Skyra-MR Avanto & 0.25 & 0.44 & 0.97 & $1.17 \pm 0.52$ \\
\hline MR Skyra-MR Symphony & 0.26 & 0.57 & 0.73 & $1.02 \pm 0.44$ \\
\hline MR Skyra-CT & 0.21 & 0.37 & 0.64 & $0.83 \pm 0.37$ \\
\hline $\begin{array}{l}\text { MR Skyra fiducial-based } \\
\text { image definition }\end{array}$ & 0.27 & 0.68 & 1.03 & $1.35 \pm 0.49$ \\
\hline \multicolumn{5}{|c|}{$\begin{array}{l}\text { Co-registration was performed for 3-T Skyra images with } 1.5-\mathrm{T} \text { Symphony, } \\
\text { 1.5-T Avanto, and CT. As a reference image, CT was chosen. Individual } \Delta \mathrm{X} \text {, } \\
\Delta \mathrm{Y} \text {, and } \Delta \mathrm{Z} \text { stereotactic coordinate deviations and total } \Delta \mathrm{R} \text { deviations }( \pm \mathrm{SD}) \\
\text { are shown. }\end{array}$} \\
\hline
\end{tabular}

definition for the MR Skyra to CT and MR Skyra to MR Avanto. The results from phantom co-registration are shown in Table 5. The co-registration deviations were also determined for the clinical case-the results of patient coregistration are shown in Table 6.

\section{Discussion}

Stereotactic definition, localization, and subsequent treatment of the target are strongly dependent on the precision of the images used. The most commonly used modality for target localization in intracranial stereotactic treatment is MRI, which is complemented by CT, digital subtraction angiography, and positron emission tomography (PET) in some situations. MRI provides excellent anatomical resolution, allows the choice of optimal pulse sequence or contrast enhancement, and minimizes artifacts caused by the stereotactic frame itself.. ${ }^{14,15,26}$ On the other hand, possible shortcomings arising from MRI in stereotactic treatment must be considered, especially geometrical distortions.

Although a substantial number of studies have dealt with the issue of MRI geometrical distortion, the topic of accuracy in image definition and co-registration still needs to be discussed and developed. Geometrical distortion in MRI usually affects the fiducial markers from the MR indicator box rather than the image itself, so fiducial distance evaluation for clinical cases can be viewed as the first step in assessing the geometrical accuracy of images. Also, comparison between MRI and CT can be performed. However, for the accurate assessment of the geometrical image distortions, direct measurement of stereotactic X, Y, and $\mathrm{Z}$ coordinates of given phantom grid points in stereotactic space is needed instead of only measurement of the distances between these points.

This study focused on comparing 3 different methods that can be used for the evaluation of image geometrical distortions of various MRI scanners while performing quality assurance. It is not very common to have the opportunity to evaluate and compare 2 commercial phantoms and 1 custom-made phantom, as in this study. The measurements were performed in the same way as for patients and thus should mimic real clinical situations very well. Evaluation was also done using clinical treatment planning software that is routinely used for patient
TABLE 6. Results of image co-registration for patient data

\begin{tabular}{lcccc}
\hline \multicolumn{1}{c}{ Patient Data } & \multicolumn{4}{c}{$\begin{array}{c}\text { Image Co-Registration Mean } \\
\text { Deviation in mm }\end{array}$} \\
\hline Co-registration & $\Delta \mathrm{X}$ & $\Delta \mathrm{Y}$ & $\Delta \mathrm{Z}$ & $\Delta \mathrm{R}$ \\
\hline MR Skyra-MR Avanto & 0.34 & 0.64 & 0.48 & $0.97 \pm 0.41$ \\
\hline MR Skyra-CT & 0.22 & 0.36 & 0.33 & $0.59 \pm 0.36$ \\
\hline $\begin{array}{c}\text { MR Skyra fiducial-based } \\
\text { image definition }\end{array}$ & 0.32 & 1.03 & 1.89 & $2.24 \pm 0.43$ \\
\hline
\end{tabular}

Co-registration was performed for 3-T Skyra images with 1.5-T Avanto and CT. As a reference image, CT was chosen. Individual $\Delta \mathrm{X}, \Delta \mathrm{Y}$, and $\Delta \mathrm{Z}$ stereotactic coordinate deviations and total $\Delta R$ deviations $( \pm S D)$ are shown.

treatment planning (Leksell GammaPlan). The presented results reflect total imaging inaccuracies, so they show not only deviation due to geometrical distortion from the MRI scanners but the inaccuracies of the whole stereotactic system (definition software, stereotactic frame).

For this study, CT images were used as a reference. There are various studies considering geometrical distortions of CT images as minimal and using CT images for MRI distortion evaluation. . $^{710,13,15,21,25}$ For the evaluation of CT imaging accuracy, the PTGR known target phantom was used. The deviations of all 21 measured crosshair coordinates from known values were calculated, and the total (radial) error of the CT scanner $\Delta \mathrm{R}$ was determined as $0.47 \pm 0.19 \mathrm{~mm}$. The distortion for each coordinate was determined as $0.10,0.30$, and $0.30 \mathrm{~mm}$ for $\mathrm{X}, \mathrm{Y}$, and $\mathrm{Z}$ coordinates, respectively. The total estimated uncertainty in distortion measurement in one coordinate was determined to be $0.32 \mathrm{~mm}$ and $0.14 \mathrm{~mm}$ for methods using and not using reference CT imaging, respectively. This result corresponds with the previously published studies ${ }^{9,13,28}$ and the determined error of the CT images was taken into account while assessing MRI results. It must also be taken into consideration that the best accuracy that can be achieved for both scanning modalities is the size of 1 pixel, which is approximately $0.5-0.9 \mathrm{~mm} .^{8}$

Results obtained from MRI and the evaluation of 3 different phantoms are presented in Table 4. Results from all 3 phantoms and methods were comparable within the level of estimated uncertainty, except for the CIRS phantom where more considerable distortion (perhaps also error of measurement) was observed in the $\mathrm{Z}$ coordinate. In general, the average distortions obtained from each individual coordinate were typically less than $0.6 \mathrm{~mm}$. However, for the CIRS phantom and $\mathrm{Z}$ coordinate, the measured distortion was larger and close to $1.0 \mathrm{~mm}$ as can be seen in Table 4 .

The total radial distortion $\Delta \mathrm{R}$ was typically lower than $0.9 \mathrm{~mm}$ for all but the CIRS phantom $(\Delta \mathrm{R}$ distortion was larger than $1.0 \mathrm{~mm}$ ).

Better results were observed for the Siemens 1.5-T Avanto and 1.5-T Symphony units than for the Siemens 3-T Skyra scanner. The results for 1.5-T units correspond with previously published studies, ${ }^{3,5,9}$ and all 3 methods presented in this study showed the capability to measure MRI geometrical distortion reliably. For the 3-T Skyra MR unit, the measured distortions were slightly higher than for the 1.5-T MR units for both the PTGR and CIRS 
phantoms; for the custom-made phantom, distortions were slightly higher for the 1.5-T Avanto and slightly lower for the 1.5-T Symphony. Our results correspond with previously published data ${ }^{1}$ and prove that geometrical distortions for $1.5 \mathrm{~T}$ and $3 \mathrm{~T}$ are comparable within the estimated error level. We also show that the distortion correction algorithm implemented in the 3-T unit suppresses the geometrical inaccuracies very well.

Image co-registration is a very valuable tool for preplanning and also patient follow-up. It also facilitates importing any nonstereotactic images (e.g., older images, PET images, etc.). By co-registration of the subsequent follow-up images with treatment images associated with treatment dose distribution, changes in lesions can be easily assessed visually. It is also a very efficient software tool in some problematic clinical situations to improve inaccuracy of distorted images. ${ }^{13,23}$

The accuracy of image co-registration was tested with the CIRS anthropomorphic phantom for 3-T Skyra images, which were co-registered to 1.5-T Avanto, 1.5-T Symphony, and CT. The 3-T Skyra MR unit was chosen for co-registration evaluation as the highest distortions for fiducial-based image definition were measured when that unit was used.

All 3 co-registered images showed better accuracy than fiducial-based image definition. Co-registration with CT images showed a total radial distortion $\Delta \mathrm{R}=0.83 \mathrm{~mm}$ compared to the fiducial-based definition of $\Delta \mathrm{R}=1.35 \mathrm{~mm}$.

The accuracy of image co-registration was also similarly tested for patient clinical data. The 3-T Skyra images were co-registered with 1.5-T Avanto and CT stereotactic template images. Both co-registered images showed better accuracy than fiducial-based 3-T Skyra image definition. Co-registration with $\mathrm{CT}$ images showed a total radial distortion $\Delta \mathrm{R}=0.59 \mathrm{~mm}$ compared to the fiducial-based definition of $\Delta \mathrm{R}=2.24 \mathrm{~mm}$. All distortions were related to $\mathrm{CT}$ as a primary reference stereotactic image. A significantly higher benefit of the co-registration compared to the fiducial-based method was observed in the case of the patient data than in the case of the CIRS phantom. The possible reason of the higher distortion measured in the patient case could be the increasing magnetic susceptibility. ${ }^{22}$ The co-registration process then shows higher improvement than in the case of inanimate phantom. The second reason for a higher distortion measured in patient data can be the use of anatomical points and landmarks, which are a greater source of uncertainty than just simple points evaluated using phantoms.

Co-registration benefits in both phantom and patient cases were obvious. Although the level of improvement in geometrical accuracy was slightly different in the phantom and patient measurements, it showed the same trend. In both cases the best geometrical accuracy was obtained for 3-T Skyra image co-registration to stereotactic CT.

Potential bias due to the definition of the CT images and using those images as reference images must be considered. However, this measurement showed the benefit of the image co-registration and the advantages of both CT scanning and MR imaging for patients to reduce the geometrical inaccuracies in the situation when MR images are more distorted.
It is, however, important to stress that co-registration itself is also associated with some imperfection and uncertainty. The best accuracy that can be achieved for image co-registration is typically considered in the range of the size of 1 pixel, which is approximately $0.5-0.9 \mathrm{~mm}{ }^{8}$ Thus, our approach is to use fiducial-based image stereotactic definition as much as possible, and only in situations (e.g., patients with shunts, metallic implants, surgical clips, etc.) when MR stereotactic images are significantly more distorted (typically over pixel size) to move to image co-registration stereotactic image definition. In such situations, stereotactic CT imaging is used as a reference/ template stereotactic image. The 3-T Skyra unit is currently not used clinically for stereotactic fiducial-based imaging at our institution, and, when necessary, images are co-registered to some other accurate stereotactic images (MRI or CT).

The results presented in this study are strictly related to a specific site and scanners used in this study. Although the study can be used as an example of observed geometrical distortions, every center must perform individual quality assurance for all imaging modalities used for stereotactic procedures.

\section{Conclusions}

The precision of both image definition by fiducials and image co-registration was investigated for $1 \mathrm{CT}$ unit and 3 MR units (two $1.5 \mathrm{~T}$ and one $3 \mathrm{~T}$ ). Three different methods were used for this study-2 commercial PTGR and CIRS phantoms and 1 custom-made cylindrical Perspex phantom. Although each method/phantom used in this study provided slightly different results, they all showed the same trend in distortion for measured MR units when considering uncertainties. All 3 methods can certainly be used for MRI distortion assessment.

The total estimated uncertainty in distortion measurement in 1 coordinate was determined to be $0.32 \mathrm{~mm}$ and $0.14 \mathrm{~mm}$ for methods using and not using reference CT imaging, respectively. Slightly larger distortions were observed when using the 3-T MR unit than when using either of the 1.5-T MR units, but all of the distortions may be comparable and are within the estimated measurement error. The maximum total radial distortion was obtained from the CIRS phantom measurement; for the 1.5-T MR unit it was $1.15 \pm 0.48 \mathrm{~mm}$, and for the 3 -T unit it was 1.35 $\pm 0.49 \mathrm{~mm}$. The co-registration process proved beneficial for geometrical distortion elimination in the case of the 3-T scanner. The best results/improvement were shown for 3-T MR image co-registration with the CT image $-0.83 \pm 0.37$ $\mathrm{mm}$ versus $1.35 \pm 0.49 \mathrm{~mm}$ for fiducial-based image definition. The co-registration results showed that the independent stereotactic imaging performed with CT or another (more accurate) MRI scanner can improve the geometrical precision of the imaging and reduce the distortion.

\section{Acknowledgments}

This work was supported by the Ministry of Health, Czech Republic-conceptual development of research organization (NHH, 00023884) projects: IG141202, IG151202-and by The Czech Science Foundation (grant 16-13323S). 


\section{References}

1. Baldwin LN, Wachowicz K, Thomas SD, Rivest R, Fallone BG: Characterization, prediction, and correction of geometric distortion in 3 T MR images. Med Phys 34:388-399, 2007

2. Computerized Imaging Reference Systems: 3D Anthropomorphic Skull Phantom, Model 603A-user guide. Publication: 603A UG 081314. Norfolk, VA: Computerized Imaging Reference Systems, 2013

3. Dammann P, Kraff O, Wrede KH, Özkan N, Orzada S, Mueller OM, et al: Evaluation of hardware-related geometrical distortion in structural MRI at 7 Tesla for image-guided applications in neurosurgery. Acad Radiol 18:910-916, 2011

4. Dean CJ, Sykes JR, Cooper RA, Hatfield P, Carey B, Swift $\mathrm{S}$, et al: An evaluation of four CT-MRI co-registration techniques for radiotherapy treatment planning of prone rectal cancer patients. Br J Radiol 85:61-68, 2012

5. Doran SJ, Charles-Edwards L, Reinsberg SA, Leach MO: A complete distortion correction for MR images: I. Gradient warp correction. Phys Med Biol 50:1343-1361, 2005

6. Foskey M, Davis B, Goyal L, Chang S, Chaney E, Strehl N, et al: Large deformation three-dimensional image registration in image-guided radiation therapy. Phys Med Biol 50:5869-5892, 2005

7. Fransson A, Andreo P, Pötter R: Aspects of MR image distortions in radiotherapy treatment planning. Strahlenther Onkol 177:59-73, 2001

8. Jursinic PA, Rickert K, Gennarelli TA, Schultz CJ: Effect of image uncertainty on the dosimetry of trigeminal neuralgia irradiation. Int J Radiat Oncol Biol Phys 62:1559-1567, 2005

9. Karger CP, Hipp P, Henze M, Echner G, Höss A, Schad L, et al: Stereotactic imaging for radiotherapy: accuracy of CT, MRI, PET and SPECT. Phys Med Biol 48:211-221, 2003

10. Mack A, Wolff R, Scheib S, Rieker M, Weltz D, Mack G, et al: Analyzing 3-tesla magnetic resonance imaging units for implementation in radiosurgery. J Neurosurg 102 (Suppl):158-164, 2005

11. Michiels J, Bosmans H, Pelgrims P, Vandermeulen D, Gybels $\mathrm{J}$, Marchal G, et al: On the problem of geometric distortion in magnetic resonance images for stereotactic neurosurgery. Magn Reson Imaging 12:749-765, 1994

12. Mizowaki T, Nagata Y, Okajima K, Kokubo M, Negoro Y, Araki N, et al: Reproducibility of geometric distortion in magnetic resonance imaging based on phantom studies. Radiother Oncol 57:237-242, 2000

13. Nakazawa H, Mori Y, Komori M, Shibamoto Y, Tsugawa $\mathrm{T}$, Kobayashi T, et al: Validation of accuracy in image coregistration with computed tomography and magnetic resonance imaging in Gamma Knife radiosurgery. J Radiat Res (Tokyo) 55:924-933, 2014

14. Novotný J Jr, Novotný J, Vymazal J, Liščák R, Vladyka V: Assessment of the accuracy of stereotactic target localization using magnetic resonance imaging: a phantom study. J Radiosurg 1:99-111, 1998

15. Novotný J Jr, Vymazal J, Novotný J, Tlachacova D, Schmitt $\mathrm{M}$, Chuda P, et al: Does new magnetic resonance imaging technology provide better geometrical accuracy during stereotactic imaging? J Neurosurg 102 (Suppl):8-13, 2005

16. Poetker DM, Jursinic PA, Runge-Samuelson CL, Wackym PA: Distortion of magnetic resonance images used in gamma knife radiosurgery treatment planning: implications for acoustic neuroma outcomes. Otol Neurotol 26:1220-1228, 2005

17. Price RR, Axel L, Morgan T, Newman R, Perman W, Schneiders N, et al: Quality assurance methods and phantoms for magnetic resonance imaging: report of AAPM Nuclear Magnetic Resonance Task Group No. 1. Med Phys 17:287-295, 1990
18. Prott FJ, Haverkamp U, Willich N, Resch A, Stöber U, Pötter R: Comparison of imaging accuracy at different MRI units based on phantom measurements. Radiother Oncol 37:221224, 1995

19. PTGR: TOPAS-Known-Target-Phantom. PTGR.de. (http:// www.ptgr.de/de/topas_known.htm) [Accessed August 3, 2018]

20. Stanescu T, Jans HS, Pervez N, Stavrev P, Fallone BG: A study on the magnetic resonance imaging (MRI)-based radiation treatment planning of intracranial lesions. Phys Med Biol 53:3579-3593, 2008

21. Stanescu T, Jans HS, Wachowicz K, Fallone BG: Investigation of a 3D system distortion correction method for MR images. J Appl Clin Med Phys 11:2961, 2010

22. Sumanaweera TS, Adler JR Jr, Napel S, Glover GH: Characterization of spatial distortion in magnetic resonance imaging and its implications for stereotactic surgery. Neurosurgery 35:696-704, 1994

23. Walker A, Liney G, Holloway L, Dowling J, Rivest-Henault D, Metcalfe P: Continuous table acquisition MRI for radiotherapy treatment planning: distortion assessment with a new extended 3D volumetric phantom. Med Phys 42:1982-1991, 2015

24. Wang D, Doddrell DM: Geometric distortion in structural magnetic resonance imaging. Curr Med Imaging Rev 1:49_ 60,2005

25. Watanabe Y, Lee CK, Gerbi BJ: Geometrical accuracy of a 3-tesla magnetic resonance imaging unit in Gamma Knife surgery. J Neurosurg 105 (Suppl):190-193, 2006

26. Weygand J, Fuller CD, Ibbott GS, Mohamed ASR, Ding Y, Yang J, et al: Spatial precision in magnetic resonance imaging-guided radiation therapy: the role of geometric distortion. Int J Radiat Oncol Biol Phys 95:1304-1316, 2016

27. Wyper DJ, Turner JW, Patterson J, Condon BR, Grossart KWM, Jenkins A, et al: Accuracy of stereotaxic localisation using MRI and CT. J Neurol Neurosurg Psychiatry 49:1445-1448, 1986

28. Yu C, Apuzzo ML, Zee CS, Petrovich Z: A phantom study of the geometric accuracy of computed tomographic and magnetic resonance imaging stereotactic localization with the Leksell stereotactic system. Neurosurgery 48:1092-1099, 2001

\section{Disclosures}

Dr. Novotný reports a consultant relationship with Elekta Instrument AB. Dr. Vymazal reports a consultant relationship with Bracco and Novocure.

\section{Author Contributions}

Conception and design: Paštyková, Novotný. Acquisition of data: Paštyková, Novotný, Veselský. Analysis and interpretation of data: Paštyková, Urgošík. Drafting the article: Paštyková. Critically revising the article: Novotný, Urgošík, Liščák. Administrative/ technical/material support: Novotný, Veselský, Liščák, Vymazal. Study supervision: Novotný, Liščák, Vymazal.

\section{Supplemental Information \\ Previous Presentations}

Portions of this work were previously presented at the 7th conference of the Czech Association of Medical Physicists, April 2017, Harrachov, Czech Republic; the 14th International Stereotactic Radiosurgery Society Congress, April 2017, Montreux, Switzerland; and the 19th Leksell Gamma Knife Society Meeting, March 2018, Dubai, United Arab Emirates.

\section{Correspondence}

Veronika Paštyková: Na Homolce Hospital, Prague, Czech Republic. veronika.pastykova@gmail.com. 